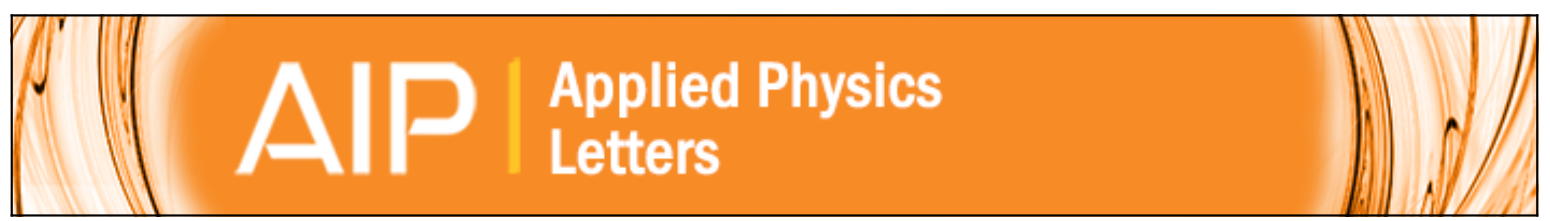

Thermoelectric power measurements of wide band gap semiconducting nanowires

Chul-Ho Lee, Gyu-Chul Yi, Yuri M. Zuev, and Philip Kim

Citation: Applied Physics Letters 94, 022106 (2009); doi: 10.1063/1.3067868

View online: http://dx.doi.org/10.1063/1.3067868

View Table of Contents: http://scitation.aip.org/content/aip/journal/apl/94/2?ver=pdfcov

Published by the AIP Publishing

Articles you may be interested in

Natural charge spatial separation and quantum confinement of $\mathrm{ZnO} / \mathrm{GaN}$-core/shell nanowires

J. Appl. Phys. 108, 123707 (2010); 10.1063/1.3504225

Contact mechanisms and design principles for (Schottky and Ohmic) metal contacts to semiconductor nanowires

J. Appl. Phys. 108, 034311 (2010); 10.1063/1.3446845

Electrical transport in GaN nanowires grown by selective epitaxy

J. Vac. Sci. Technol. B 27, 2040 (2009); 10.1116/1.3123302

Surface depletion effects in semiconducting nanowires

J. Appl. Phys. 103, 104313 (2008); 10.1063/1.2932072

Electrical properties and near band edge emission of Bi-doped $\mathrm{ZnO}$ nanowires

Appl. Phys. Lett. 90, 083113 (2007); 10.1063/1.2431715

Want to publish your paper in the \#1 MOST CITED journal in applied physics?

With Applied Physics Letters, you can.

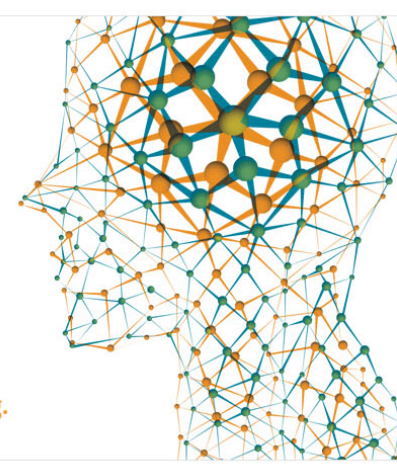




\title{
Thermoelectric power measurements of wide band gap semiconducting nanowires
}

\author{
Chul-Ho Lee, ${ }^{1}$ Gyu-Chul Yi, ${ }^{2, a)}$ Yuri M. Zuev, ${ }^{3}$ and Philip Kim ${ }^{3, b)}$ \\ ${ }^{1}$ National Creative Research Initiative Center for Semiconductor Nanorods and Department of Materials \\ Science and Engineering, POSTECH, Pohang, Gyeongbuk 790-784, Republic of Korea \\ ${ }^{2}$ Department of Physics and Astronomy, Seoul National University, Seoul 151-747, Republic of Korea \\ ${ }^{3}$ Department of Applied Physics and Applied Mathematics and Department of Physics, Columbia University, \\ New York, New York 10027, USA
}

(Received 21 September 2008; accepted 16 December 2008; published online 13 January 2009)

We investigated the temperature-dependent thermoelectric power (TEP) of individual wide band gap $\mathrm{ZnO}$ and $\mathrm{GaN}$ semiconducting nanowires by fabricating the devices with good Ohmic contacts. In the temperature range of 10-300 K, the measured TEP of both nanowires was linearly dependent on temperature, indicating the degenerate doping nature of these nanowires. The room temperature TEP value of $\mathrm{ZnO}$ nanowires was as high as $-400 \mu \mathrm{V} / \mathrm{K}$ while an order of magnitude smaller TEP value was observed in GaN. The negative sign of TEP values shows that electrons are the majority carriers in these wide band gap nanowires. More importantly, in comparison with gate-dependent transport measurements of the nanowire field effect transistors, analysis of temperature-dependent TEP measurements provides a reliable way of estimating the majority carrier concentration of nanowires, where conventional Hall effect measurements cannot be used. (C) 2009 American Institute of Physics. [DOI: 10.1063/1.3067868]

Wide band gap semiconducting nanowires such as $\mathrm{ZnO}$ and $\mathrm{GaN}$ have provided potential candidates for building blocks for short wavelength optoelectronics, transparent electronics, and high-temperature electronics applications. ${ }^{1-3}$ Central to these applications is to control and measure the doping concentration of the majority carriers. Due to the confined geometry of the one-dimensional (1D) nanostructure, however, determination of the carrier concentration by a traditional Hall effect measurement is not possible. Alternatively, the estimation of carrier concentration using the field effect transport measurement suffers from the ill-defined charge traps in the dielectric and oxide layers. ${ }^{4}$ In this study, we present a reliable method to determine the majority carrier concentration in wide band gap semiconducting nanowires employing temperature-dependent thermoelectric power (TEP) measurements. TEP and electrical conductance were measured for $\mathrm{ZnO}$ and $\mathrm{GaN}$ nanowires in a field effect transistor (FET) device geometry with a microfabricated heater. The observed TEP, showing linear dependence on temperature, allows us to measure the majority carrier concentration in these wide band gap nanowires using the Mott formula. ${ }^{10}$

Recently the potential applications for thermoelectrics that employ semiconducting nanowires have recently emerged. ${ }^{5,6}$ TEP, the ratio of the electric field to the thermal gradient, is one of the central physical quantities that determine the efficiency of thermoelectric applications. In general, TEP of an electronic system depends on the electron and hole asymmetries at the Fermi level, and thus is sensitive to small changes in scattering rate and the density of states at the Fermi level. ${ }^{7}$ Utilizing this sensitivity, the electrical properties of 1D nanostructures, such as carbon nanotubes (CNTs) and semimetallic Bi nanowires, have been investi-

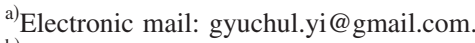

${ }^{b)}$ Electronic mail: pk2015@columbia.edu.
}

gated by TEP measurements. ${ }^{8,9,11}$ In addition, TEP measurements have provided an effective approach to measure carrier concentration in nanowires. ${ }^{12,13}$ In order to extend this mesoscopic TEP measurement scheme to individual wide band gap semiconducting nanowires, it is necessary to fabricate devices with Ohmic-like low contact resistance. This often poses an experimental challenge due to the presence of a Schottky barrier at the contact area. In this study, we demonstrate that Ohmic-like contacts can be fabricated in $\mathrm{ZnO}$ and $\mathrm{GaN}$ nanowires with a proper plasma treatment on the contact area prior to the metal evaporation. This process creates a thin Schottky barrier at the junctions, allowing simultaneous electrical and TEP measurements of individual $\mathrm{ZnO}$ and $\mathrm{GaN}$ nanowires in the temperature range of $10-300 \mathrm{~K}$.

A schematic layout of a typical device for mesoscopic electrical and TEP measurements is similar to that of our previous studies to measure TEP in CNTs. ${ }^{8,9}$ As shown in Fig. 1, the nanowire-hybrid device used in this study consists of one microfabricated heater and two sets of thermometers

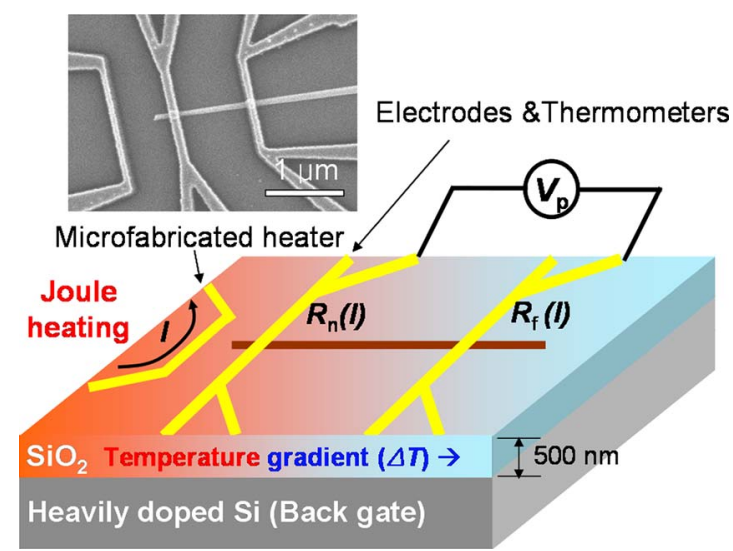

FIG. 1. (Color online) Schematic layout and scanning electron microscopy image of a nanowire device for TEP measurements. 

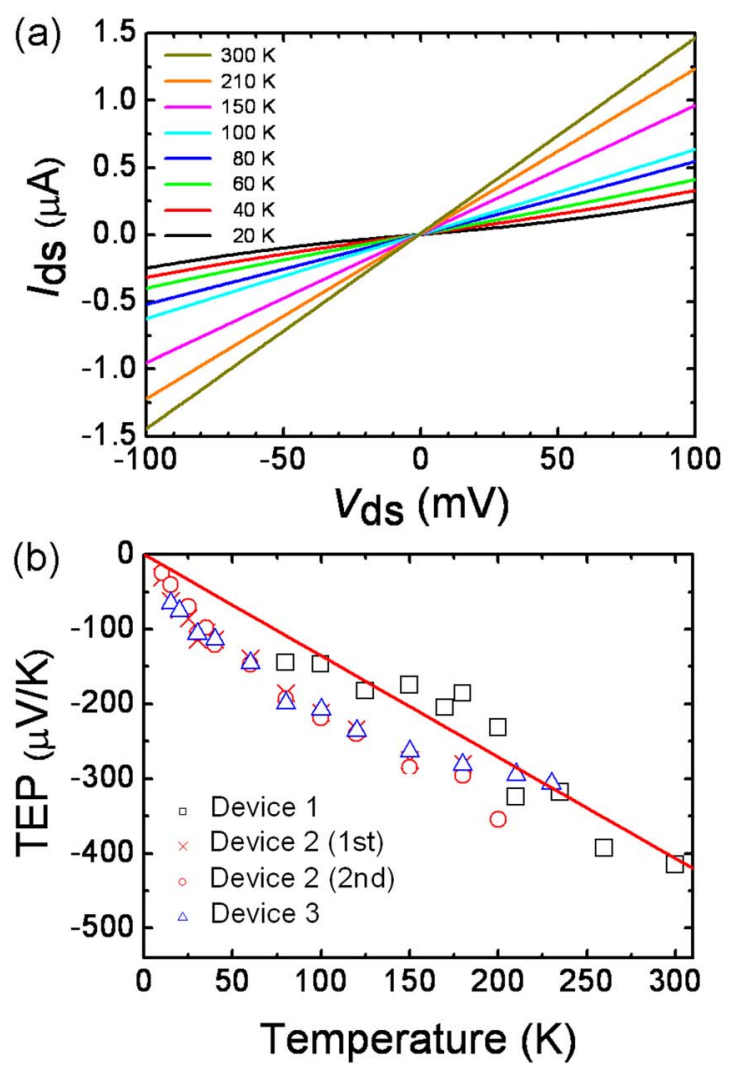

FIG. 2. (Color online) (a) Typical $I_{\mathrm{ds}}-V_{\mathrm{ds}}$ characteristics of $\mathrm{ZnO}$ nanowire devices as a function of temperature. Symmetric and linear $I_{\mathrm{ds}}-V_{\mathrm{ds}}$ behavior down to $80 \mathrm{~K}$ and nonlinear behavior in the low-bias region below $80 \mathrm{~K}$ indicates the formation of low and narrow Schottky barriers near the contact regions. (b) TEP values of individual $\mathrm{ZnO}$ nanowires as a function of temperature. The solid line is a linear fit to zero with the slope of $-1.36 \pm 0.05 \mu \mathrm{V} / \mathrm{K}^{2}$.

which simultaneously serve as electrodes for electrical measurements of the nanowires. The microfabricated heater and electrodes were defined using standard electron beam lithography, followed by metal deposition and lift-off processes. The details of the measurement technique were explained elsewhere. ${ }^{8}$ Briefly, by applying current to the heater, a temperature gradient $(\Delta T)$ can be built up along the nanostructures via Joule heating of the microheater, which was measured by probing the changes of the four-probe resistance in the near and far thermometers, $R_{n}$ and $R_{f}$, respectively. The thermoelectric voltage $\left(\Delta V_{p}\right)$ corresponding to $\Delta T$ across the nanostructures was also measured and the TEP was consequently obtained by the simple relation $S=-\Delta V_{p} / \Delta T$.

We now discuss the results on $\mathrm{ZnO}$ nanowires which were grown by catalyst-free metal-organic vapor-phase epitaxy. ${ }^{14}$ Three different devices with the nanowire diameter in the range of 80-120 $\mathrm{nm}$ and a channel length of $\sim 1 \mu \mathrm{m}$ were fabricated in this study. Figure 2(a) shows a typical drain current $\left(I_{\mathrm{ds}}\right)$ versus source-drain bias voltage $\left(V_{\mathrm{ds}}\right)$ characteristic at various temperatures. The linear and symmetric $I_{\mathrm{ds}}-V_{\mathrm{ds}}$ characteristics are shown in the temperature range of 80-300 K, suggesting minimal influence of a junction barrier between electrodes and the $\mathrm{ZnO}$ nanowire. A typical contact resistance estimated from a separate fourterminal measurement is in the range of $10-80 \mathrm{k} \Omega$ at room temperature. This low contact resistance suggests that the Schottky barrier is narrow and thus the measured TEP reflects the contribution from the bulk part of the nanowires

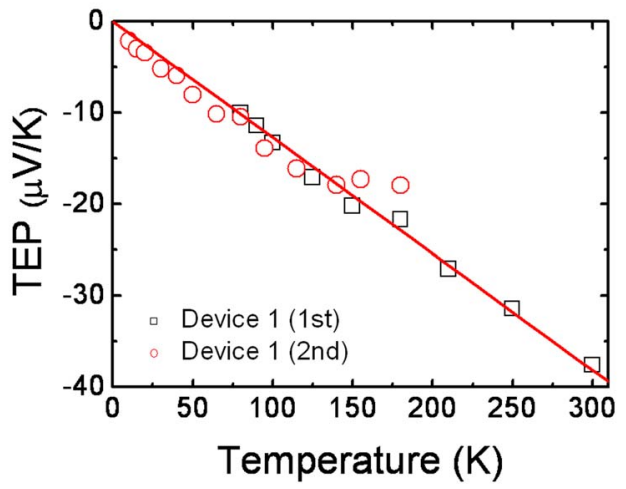

FIG. 3. (Color online) TEP values of individual GaN nanowires as a function of temperature. The slope of the linear fit to zero is $-0.127 \pm 0.002 \mu \mathrm{V} / \mathrm{K}^{2}$

and not the depletion regions for $T>80 \mathrm{~K}$. In the temperature ranges below $80 \mathrm{~K}$, however, nonlinear $I_{\mathrm{ds}}-V_{\mathrm{ds}}$ behavior started to appear in the low-bias region, implying increasing effect of the Schottky barriers in transport.

Figure 2(b) shows the measured TEP in the temperature range of $10-300 \mathrm{~K}$ for three different $\mathrm{ZnO}$ devices. The measured TEP $(S)$ was about $-400 \mu \mathrm{V} / \mathrm{K}$ at room temperature, varying linearly with temperature. The negative sign of TEP values indicates that the carrier transport in $\mathrm{ZnO}$ nanowires is contributed by electron diffusion, reflecting that the nanowires are $n$-type semiconductors. ${ }^{7}$ The overall temperature dependence, $S \propto T$, agrees well with the Mott relation expected for a metal or a heavily doped semiconductor. ${ }^{7}$ From the linear fit of TEP to temperature, we obtain an average slope of $-1.36 \pm 0.05 \mu \mathrm{V} / \mathrm{K}^{2}$. We will later discuss that this value can be related to the carrier density in the nanowire.

Figure 3 shows the simultaneously measured conductance and TEP of individual Ni-catalyzed $\mathrm{GaN}$ nanowires with a diameter of $\sim 80 \mathrm{~nm}$. Similar to the $\mathrm{ZnO}$ nanowire experiments, the linear $I_{\mathrm{ds}}-V_{\mathrm{ds}}$ characteristic curves were observed at higher temperatures, subsequently turning into more nonlinear behavior at lower temperatures due to the formation of Schottky barriers at the electrode interface. The measured TEP value of GaN was also all negative in the entire temperature range. We note that, however, $|S|$ $\approx 40 \mu \mathrm{V} / \mathrm{K}$ at room temperature, which is one order of magnitude smaller than $|S|$ of $\mathrm{ZnO}$ nanowires. The temperature-dependent TEP exhibited the linear dependence, $S \propto T$, with the slope of $-0.127 \pm 0.002 \mu \mathrm{V} / \mathrm{K}^{2}$.

The major experimental result in the above measurements is that absolute values of TEP for the wide band gap semiconductors increase linearly with temperature. This linear dependence, observed in both $\mathrm{ZnO}$ and $\mathrm{GaN}$ nanowires, suggests that there is no appreciable phonon-drag induced TEP in these nanowires, and thus TEP can be described by thermal diffusion of the majority carriers. In addition, this slope can be related to the carrier concentration in the nanowire channels. Carrier concentration for semiconductor nanowires is often difficult to be determined accurately due to the confined geometry in which the Hall effect measurement is not applicable due to the experimental challenge to fabricate a suitable Hall bar geometry. A traditional way of carrier concentration estimation employing gate-dependent field effect transport ${ }^{15}$ often suffers from lack of accurate determination of the capacitance coupling and the trapped charge densities. As we demonstrate below the TEP mea- 


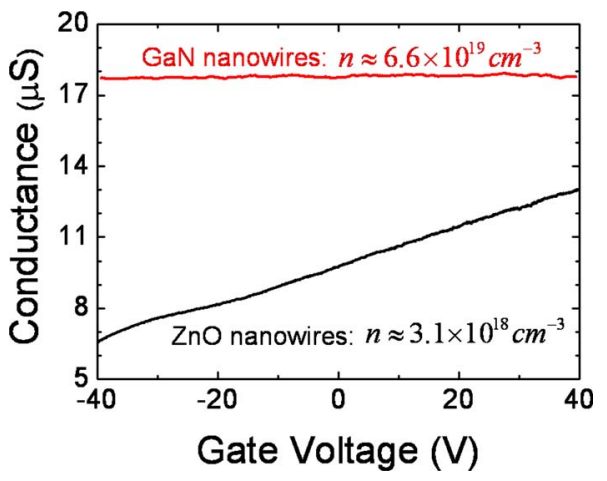

FIG. 4. (Color online) Gate-dependent conductance characteristics of $\mathrm{ZnO}$ and $\mathrm{GaN}$ nanowire devices at room temperature. No appreciable gate dependence in conductance of GaN nanowire devices indicates high carrier concentration in the sample. The indicated carrier densities $n$ were estimated from TEP measurements.

surement can be used to estimate the carrier concentration using the semiclassical Mott relation ${ }^{10}$

$$
S=-\left.\frac{\pi^{2}}{3} \frac{k^{2} T}{|e|} \frac{d \ln \sigma}{d E}\right|_{E=E_{f}}=-\frac{\pi^{2} k^{2} m^{*}}{\left(3 \pi^{2}\right)^{2 / 3} \hbar^{2}|e|} \frac{T}{n^{2 / 3}} .
$$

Here, the right hand part of the equation was derived by assuming an energy-independent mobility ${ }^{16}$ where $n$ is the carrier concentration and $m^{*}$ is the effective mass of the material. We also note that Eq. (1) can be valid for our samples because their mean free path, $l_{m} \geq 3 \mathrm{~nm}$, is much larger than the interatomic distance and that this semiclassical approach is appropriate without a quantum correction since $k_{F} l_{m} \sim 1$, where $k_{F}$ is the Fermi wave vector. Similar approximation has been made in the TEP analysis of semimetallic $\mathrm{Bi}$ nanowires ${ }^{17}$ and small gap semiconductors. ${ }^{13}$ Applying Eq. (1) to the experimentally obtained linear slope of $1.36 \mu \mathrm{V} / \mathrm{K}^{2}$ for $\mathrm{ZnO}$ nanowires, we obtain $n \approx 3.1$ $\times 10^{18} \mathrm{~cm}^{-3}$ for the $\mathrm{ZnO}$ nanowires assuming the effective mass $m^{*} \approx 0.28 m_{e}$ from bulk $\mathrm{ZnO}{ }^{18}$ Similarly, using the slope value of $0.127 \mu \mathrm{V} / \mathrm{K}^{2}$ and the bulk effective mass $m^{*} \approx 0.20 m_{e},{ }^{19}$ we estimate $n \approx 6.6 \times 10^{19} \mathrm{~cm}^{-3}$ for $\mathrm{GaN}$ nanowires, which is an order of magnitude higher than that of $\mathrm{ZnO}$ nanowires.

We now compare the estimated carrier concentrations with those determined from the gate-dependent field effect transport measurements at room temperature in Fig. 4. For $\mathrm{ZnO}$ nanowire devices, as gate bias voltage $\left(V_{g}\right)$ increases, the conductance also increases, indicating that the major carriers are electrons, in accordance with the previous report, ${ }^{20}$ which agrees with the results of TEP measurement on the same nanowires. In particular, the substantial increase in conductance with increasing $V_{g}$ suggests that $n$ is comparable to the field effect induced charge $n_{\mathrm{FE}} \approx 5.7$ $\times 10^{18} \mathrm{~cm}^{-3}$, which is calculated from the capacitance coupling based on the device geometric consideration. This value agrees reasonably with the estimated $n$ from the TEP measurement, considering that the field effect induced charge can overestimate the carrier concentration due to charge trapping. On the other hand, GaN nanowire devices exhibited almost complete metallic behavior, showing no appreciable gate dependence within experimentally accessible gate voltage range. Considering gate coupling in this device geometry, for the applied gate voltage range, -40 to $40 \mathrm{~V}$, the accessible charge density $n_{\text {acc }}$ is estimated to be $n_{\text {acc }} \approx 3.8$ $\times 10^{18} \mathrm{~cm}^{-3}$. The absence of gate dependence in the GaN nanowire FET indicates that the carrier concentration is much larger than $n_{\text {acc }}$, in accordance with the TEP measurement where the estimated majority carrier density indeed satisfies $n \gg n_{\text {acc. }}$. While FET characteristics provide an unreliable value of the carrier concentration of highly doped wide band gap semiconductors, such as $\mathrm{ZnO}$ and $\mathrm{GaN}$ nanowires studied here, TEP measurements provide an excellent tool to measure carrier concentration experimentally.

In conclusion, we performed electrical and TEP measurements of individual $\mathrm{ZnO}$ and $\mathrm{GaN}$ nanowires using a mesoscopic measurement technique in the temperature range of 10-300 K. The measured TEP increases linearly with temperature for both nanowires, exhibiting a TEP of $-400 \mu \mathrm{V} / \mathrm{K}$ for $\mathrm{ZnO}$ nanowires which is larger than that of $\mathrm{GaN}$ by an order of magnitude at room temperature. The negative sign of TEP values indicates that the majority carriers of these nanowires are electrons. Employing the Mott relation, we demonstrate that the carrier concentration can be obtained from the temperature-dependent TEP measurements in conjunction with conductance measurements, even when the conventional field effect estimation is not possible.

This work was financially supported by the National Creative Research Initiative Project (Grant No. R16-2004004-01001-0) of the Korea Science and Engineering Foundations (KOSEF), MRSEC Program of the NSF (Grant No. DMR-02-13574), and NSF CAREER (Grant No. DMR0349232).

${ }^{1}$ C. M. Lieber and Z. L. Wang, MRS Bull. 32, 99 (2007).

${ }^{2}$ G.-C. Yi, C. Wang, and W. I. Park, Semicond. Sci. Technol. 20, S22 (2005).

${ }^{3}$ P. J. Pauzauskie and P. Yang, Mater. Today 9, 36 (2006).

${ }^{4}$ S. A. Dayeh, C. Soci, P. K. L. Yu, E. T. Yu, and D. Wang, Appl. Phys. Lett. 90, 162112 (2007).

${ }^{5}$ A. I. Boukai, Y. Bunimovich, J. Tahir-Kheli, J.-K. Yu, W. A. Goddard III, and J. R. Heath, Nature (London) 451, 168 (2008).

${ }^{6}$ A. I. Hochbaum, R. Chen, R. D. Delgado, W. Liang, E. C. Garnett, M. Najarian, A. Majumdar, and P. Yang, Nature (London) 451, 163 (2008).

${ }^{7}$ D. K. C. Macdonald, Thermoelectricity (Dover, New York, 2006).

${ }^{8}$ J. P. Small, L. Shi, and P. Kim, Solid State Commun. 127, 181 (2003).

${ }^{9}$ J. P. Small, K. M. Perez, and P. Kim, Phys. Rev. Lett. 91, 256801 (2003).

${ }^{10}$ M. Cutler, J. F. Leavy, and R. L. Fitzpatrick, Phys. Rev. 133, A1143 (1964)

${ }^{11}$ A. Boukai, K. Xu, and J. R. Heath, Adv. Mater. (Weinheim, Ger.) 18, 864 (2006).

${ }^{12}$ Y.-M. Lin, O. Rabin, S. B. Cronin, J. Y. Ying, and M. S. Dresselhaus, Appl. Phys. Lett. 81, 2403 (2002).

${ }^{13}$ F. Zhou, J. Szczech, M. T. Pettes, A. L. Moore, S. Jin, and L. Shi, Nano Lett. 7, 1649 (2007).

${ }^{14}$ W. I. Park, D. H. Kim, S.-W. Jung, and G.-C. Yi, Appl. Phys. Lett. 80 4232 (2002)

${ }^{15}$ R. Martel, T. Schmidt, H. R. Shea, T. Hertel, and P. Avouris, Appl. Phys. Lett. 73, 2447 (1998).

${ }^{16}$ From gate-dependent FET behavior, we found that the field effect mobility is constant, and thus the energy-dependent change in mobility is negligible in the experimental range.

${ }^{17}$ J. Heremans and C. M. Thrush, Phys. Rev. B 59, 12579 (1999).

${ }^{18}$ A. Ohtomo, M. Kawasaki, I. Ohkubo, H. Koinuma, T. Yasuda, and Y. Segawa, Appl. Phys. Lett. 75, 980 (1999).

${ }^{19}$ S. N. Mohammad and H. Morkoç, Prog. Quantum Electron. 20, 361 (1996).

${ }^{20}$ H.-J. Kim, C.-H. Lee, D.-W. Kim, and G.-C. Yi, Nanotechnology 17, S327 (2006) 